\title{
Ultraviolet-based bonding for perfluoropolyether low aspect-ratio microchannels and hybrid devices $\uparrow$
}

\author{
Carmela De Marco, ${ }^{a, b}$ Salvatore Girardo, ${ }^{a, b}$ Elisa Mele, ${ }^{a}$ Roberto Cingolani ${ }^{a}$ and Dario Pisignano ${ }^{a, b}$
}

\author{
Received 25th February 2008, Accepted 6th May 2008 \\ First published as an Advance Article on the web 11th June 2008 \\ DOI: 10.1039/b803243b
}

\begin{abstract}
Producing solvent-resistant microfluidic devices is a challenge for analytical chemistry and biochemistry. We demonstrate a simple and low-cost fabrication approach for the realization of solvent-resistant microchannels based on perfluoropolyether elastomers, exhibiting very low aspect ratios (0.01). The strength of the microchannels sealing is evaluated through the maximum internal pressure (1.52 $\mathrm{MPa}$ ) prior to device failure, due to delamination at the bonded interface. This approach allows the elastic properties of silicone elastomers, suitable for high quality external connections, to be combined with the non-swelling character of perfluoropolyethers.
\end{abstract}

\section{Introduction}

The basic element of microfluidic devices is a network of hydraulically connected microchannels. This is typically obtained by the irreversible sealing between two surfaces, i.e. a surface embedding a pattern of fluidic capillaries, and a second element working as a flat cap. Some important challenges relate to such assembly, namely: (i) avoiding the deformation of the microand nanofeatures and the eventual channel clogging during the sealing procedure; (ii) assuring good adhesion between the two surfaces for producing watertight channels able to support high internal pressure (up to the order of several MPa) ${ }^{1}$ (iii) effectively interfacing the resulting device with external fluid pumping systems. In this framework, polymers are widely chosen materials, being characterized by easy and inexpensive manufacturability, biocompatibility, good thermal resistance, and optical transparency. They can be processed by various methods, such as high temperature imprinting, ${ }^{2-4}$ laser assisted molding ${ }^{5}$ and ablation, ${ }^{6}$ in situ surface polymerization assisted by ultraviolet (UV), thermal, ${ }^{7}$ or redox initiation, ${ }^{8}$ injection molding ${ }^{9,10}$ and soft lithography, ${ }^{11-15}$ enabling low cost and highvolume production of disposable chips. ${ }^{12-14}$ In particular, various assembly and bonding methods have been reported, depending on the specific polymers, allowing one to mutually seal surfaces made by the same compound, or by materials with different chemico-physical properties.

To date, the most used polymers in nano- and microfluidics include polymethylmethacrylates (PMMAs) and cycloolefin copolymers (COCs) as thermoplastic materials, and polydimethylsiloxane (PDMS), among elastomers. Their irreversible sealing can be accomplished by means of thermal ${ }^{16-19}$

${ }^{a} N N L$, National Nanotechnology Laboratory of CNR-INFM, Università del Salento c/o Distretto Tecnologico, and Italian Institute of

Technology, via Arnesano, I-73100, Lecce, Italy.

E-mail: Dario.pisignano@unile.it; Fax: +390832298146

${ }^{b}$ Scuola Superiore ISUFI, Università del Salento, via Arnesano, I-73100, Lecce, Italy

$\dagger$ The HTML version of this article has been enhanced with colour images. or UV/ozone and oxygen plasma treatments, ${ }^{11,20}$ solvents, ${ }^{4}$ and gluing layers. ${ }^{21,22}$ However, these polymers and their derivatives present some serious limitations for chemical and biological microfluidic applications involving non-polar organic solvents, because of their solubility (PMMA and COCs) ${ }^{23}$ or swelling behavior (PDMS) ${ }^{24}$ Furthermore, in the bonding procedures usually applied to rigid thermoplastic polymers, such as PMMA or COCs, the contact between surfaces is assisted by external pressures $\left(10^{2}-10^{4} \mathrm{kPa}\right)$ combined with thermal and surface treatments. ${ }^{4,1920,25}$ This may lead to a remarkable reduction of the channel height in the final microfluidic chips, and to deformations of the microstructures up to the occlusion of the assembled capillaries. ${ }^{1,16,20,25}$

An interesting alternative class of polymers is represented by perfluoropolyether (PFPE) elastomers, ${ }^{26}$ whose application to microfluidic chips has been pioneered by DeSimone and coworkers with a PFPE-urethane-methacrylate. ${ }^{26}$ These materials exhibit some appealing properties of PDMS (transparency, flexibility, and conformability) together with absence of swelling and enhanced structural stability, ${ }^{27,28}$ however their bonding to surfaces can be made difficult by their intrinsic unfouling character. Moreover, using elastomers with a lower Young's modulus facilitates the connections to external tubing. Therefore, the implementation of effective bonding procedures for PFPE compounds within easily interconnected hybrid devices is important for a full exploitation of the material performances in lab-on-chips.

In this work, we report on a UV-based bonding process by a photocurable PFPE-urethane-methacrylate with low molecular weight into a hybrid polymer chip. This PFPE grade exhibits the typical oleophobicity, solvent resistance, and mechanical stability of fluoroelastomers. Hybrid PFPE/PDMS micropatterned elements are here realized, consisting of a thin fluoroelastomer layer (thickness $\sim 3 \mu \mathrm{m})$ and a thick $(\sim 3 \mathrm{~mm}$ ) backside element of PDMS. In particular, we demonstrate irreversible sealing between two PFPE surfaces without losses in the microchannel height, which allows one to lithographically realize capillaries with very low aspect ratio value (0.01) without clogging, and stable up to pressures of 1.52 $\mathrm{MPa}$. This approach guarantees 
both wide solvent compatibility and effective connections of the microfluidic chip for fluid injection and control.

\section{Experimental}

\section{Materials}

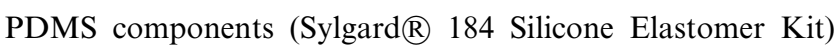
are purchased from Dow Corning (Midland, MI), and the PFPE prepolymer used in this work is kindly provided by Solvay-Solexis (Bollate, Italy). With respect to fluorinated oligomers with acryloxy terminations, recently employed for the fabrication of hybrid stamps for soft lithographies, ${ }^{29}$ this compound shows methacryloxy ending groups, almost double molecular weight and, importantly, lower surface energy, which is important for the faithful replication of master templates. By contact angle measurements, we found a surface energy as low as $14.9 \mathrm{mN} \mathrm{m}^{-1}$.

The PDMS prepolymer is obtained by mixing the base and curing agent in a $9: 1 \mathrm{w} / \mathrm{w}$ ratio. The photoinitiator, Darocur $\AA$ 1173, is supplied by Ciba (Pontecchio Marconi, Italy). The photoresist, AZ5214E, and its developer are purchased from Clariant Corporation's AZ Electronic Materials (Wiesbaden, Germany), and $\mathrm{Si}$ and $\mathrm{Si} / \mathrm{SiO}_{2}$ wafers, with oxide thickness $\sim 100 \mathrm{~nm}$, are purchased from Si-Mat (Landsberg, Germany). Capillary tubing connections (Tygon $($ ) are purchased from Norton Performance Plastics (Akron, OH). The employed chemical reagents $\left(\mathrm{NH}_{4} \mathrm{~F}, \mathrm{HF}, \mathrm{KOH}\right)$ are purchased from Sigma-Aldrich (Milan, Italy). Chloroform and toluene are from J. T. Baker (Milan, Italy).

\section{Composite PDMS/PFPE molds}

The first step for realizing the microfluidic network is the fabrication of a master template by standard photolithography. The photoresist is deposited onto a $\mathrm{Si}-\mathrm{SiO}_{2}$ substrate by spincoating at $4000 \mathrm{rpm}$ for $40 \mathrm{~s}$, and exposed to UV radiation for $3 \mathrm{~min}$ at $45 \mathrm{~W}$. After development for $1 \mathrm{~min}$ and substrate rinsing, the oxide is etched by $\mathrm{NH}_{4} \mathrm{~F} / \mathrm{HF} / \mathrm{H}_{2} \mathrm{O}(6.6 \mathrm{~g} / 1.6 \mathrm{ml} / 10 \mathrm{ml})$, and $2 \mu \mathrm{m}(h)$ of $\mathrm{Si}$ are removed by a $5.9 \mathrm{M} \mathrm{KOH}$ solution at $110{ }^{\circ} \mathrm{C}$ for $20 \mathrm{~s}$. The pattern includes a $1 \mathrm{~cm}$ long capillary of width $(W) 200 \mu \mathrm{m}$, connected with lateral reservoirs as fluid inlet and outlet. Two lateral rulers are integrated in the chip, having squared ticks $\left(100 \times 100 \mu \mathrm{m}^{2}\right)$ separated by $100 \mu \mathrm{m}$, useful for the accurate investigation of the fluid motion.

The master geometry is replicated by photocurable PFPEurethane-methacrylate. A $4 \% \mathrm{w} / \mathrm{w}$ solution of PFPE prepolymer and photoinitiator is prepared by mixing the two components and degassing the mixture at room temperature in a vacuum. The solution is deposited onto the master surface by spincoating at $4000 \mathrm{rpm}$ for $40 \mathrm{~s}$ (Fig. 1(a)), and cured under UV irradiation for $30 \mathrm{~s}\left(\lambda \cong 360 \mathrm{~nm}\right.$; intensity $\left.=6 \mathrm{~mW} \mathrm{~cm} \mathrm{~cm}^{-2}\right)$ under nitrogen atmosphere to prevent the reaction between the photoinitiator and oxygen (Fig. 1(b)). The thickness of the resulting PFPE layer on the master surface is about $3 \mu \mathrm{m}$. For obtaining composite PFPE/PDMS molds, a thick layer $(\sim 3 \mathrm{~mm})$ of PDMS is poured onto the fluoroelastomer film, and the subsequent polymerization is carried out at $70{ }^{\circ} \mathrm{C}$ for $2 \mathrm{~h}$ (Fig. 1(c)). The textured PFPE/PDMS element is then peeled off from the master (Fig. 1(d)), and fluid access ports are opened
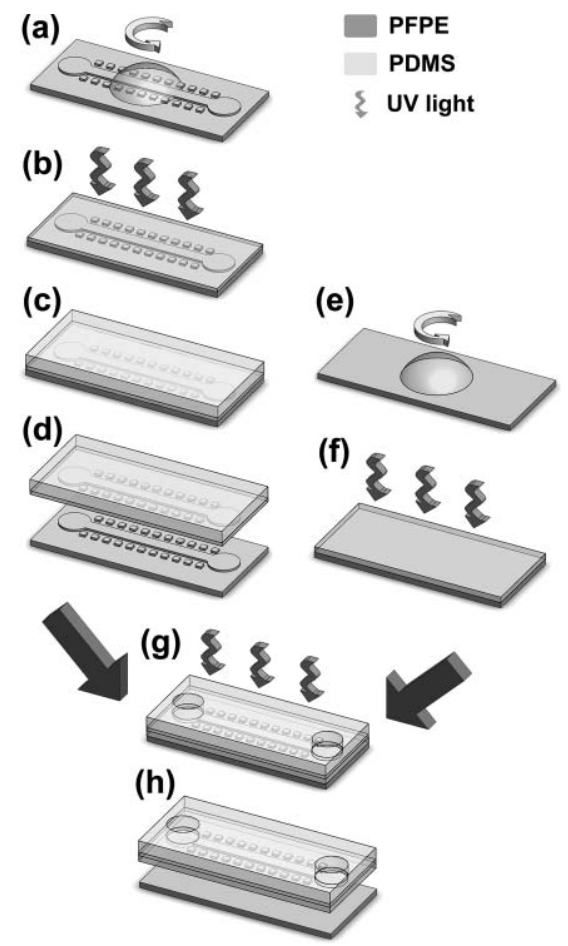

Fig. 1 Scheme of chip fabrication. Spin-coating of PFPE solution onto the master surface (a), PFPE UV curing (b), PDMS pouring (c), peelingoff the hybrid PFPE/PDMS mold (d), spin-coating of PFPE solution (e); PFPE partial curing (f), bonding (g), and chip separation (h).

corresponding to the reservoirs by carefully producing $0.75 \mathrm{~mm}$ diameter holes with a core sample cutter.

A thin PFPE slab ( $\cong 60 \mu \mathrm{m})$ working as chip cap is realized by spin-coating (350 rpm for $25 \mathrm{~s}$ ) onto a Si wafer (Fig. 1(e)), and UV curing for $20 \mathrm{~s}$ under nitrogen (Fig. 1(f)). The polymerization time is slightly shorter than the exposure interval used for PDMS/PFPE mold, in order to provide a partial curing of the fluoroelastomer, which is important for then obtaining a good, irreversible sealing. At this stage, the PFPE slab is not peeled off from the Si substrate.

\section{Bonding and device connection}

To assemble the sealed microfluidic device, the composite PDMS/PFPE mold is placed on the PFPE slab, which results in the conformal contact between the two exposed fluoroelastomer surfaces. The system is then irradiated with UV light for a few minutes under nitrogen (Fig. 1(g)), thus completing the polymerization of the PFPE flat slab and resulting in the bonding between the two elements. Finally, the whole plastic chip is separated from Si (Fig. 1(h)).

The microfluidic chip is connected to a syringe infusion pump (Harvard Apparatus, Holliston, MA, USA) for liquid injection and control. Plastic tubes (id $=0.25 \mathrm{~mm}$, od $=0.78 \mathrm{~mm}$ ) are fitted manually in the inlet and outlet holes without causing delamination at the PDMS/PFPE and PFPE/PFPE interfaces, the elastic properties of the silicone elastomer guaranteeing stable and watertight connections. ${ }^{15}$ The free end of the tubes is coupled to the stainless steel needle $(27 \mathrm{G})$ of $1 \mathrm{ml}$ syringes. 


\section{Leakages tests}

To quantitatively determine the bond strength of the assembled devices, deionized water injection is carried out at growing flow rates from 5 to $45 \mu \mathrm{l} \mathrm{min} \mathrm{mith}^{-1}$ increments of $5 \mu 1 \mathrm{~min}^{-1}$, up to delamination at the sealed interfaces. In particular, for each applied flow rate the fluid dynamics into the microchannel is observed by an optical microscope through a high-speed camera (FastCam APX-RS, Photron, CA) for about $1 \mathrm{~min}$. The exact position of the fluid front along the microchannel during filling at room temperature is determined by an acquisition rate of 125 frames $\mathrm{s}^{-1}$ by a dedicated viewer software. After each experiment, the device is placed in an oven for $2 \mathrm{~h}$ at $70{ }^{\circ} \mathrm{C}$, to allow the complete evaporation of residual water in the microchannels. Afterwards, the flow rate is increased and a new filling experiment is performed on the same device.

Filling experiments are also carried out by both chroloform and toluene (flow rate $=5 \mu 1 \mathrm{~min}^{-1}$ ) to test the device resistance against organic solvents. The distance between the connecting plastic tubes and the PFPE microchannel ranges from a few hundreds of $\mu \mathrm{m}$ to $1 \mathrm{~mm}$, which means that injected organic solvents can undergo partial contact with PDMS. However, during filling experiments we can not appreciate any PDMS/PFPE delamination due to eventual local PDMS swelling domains.

\section{Results}

Fluoroelastomer molds made by the PFPE compound used here can exhibit a poor conformal contact when having thickness above $70 \mu \mathrm{m}$. Since for fabricating and tightly interconnecting microfluidic chips one needs polymeric elements with typical thicknesses of a few millimeters, hybrid PFPE-urethanemethacrylate/PDMS molds are preferable. In fact, they combine excellent conformability to the counterfacing PFPE slab, good fluidic functionality of the resulting microchannel network in terms of solvent resistance, and PDMS elasticity for firmly fitting capillary tubing to access holes. ${ }^{15}$ The resulting polymer microchannels (with cross section, $W \times h=200 \times 2 \mu \mathrm{m}^{2}$ ) exhibit an aspect ratio $(h / W)$ as low as 0.01 (Fig. 2), impossible to be achieved by silicone elastomers, for which $h$ values below a few tenths of $W$ determine feature collapse and sagging upon conformal contact. ${ }^{30}$ We point out that microchannels with very low aspect ratios are relevant to many lab-on-chip and diagnostic applications, allowing one to have uniform conditions of optical excitation of flowing chromophores and access to the whole

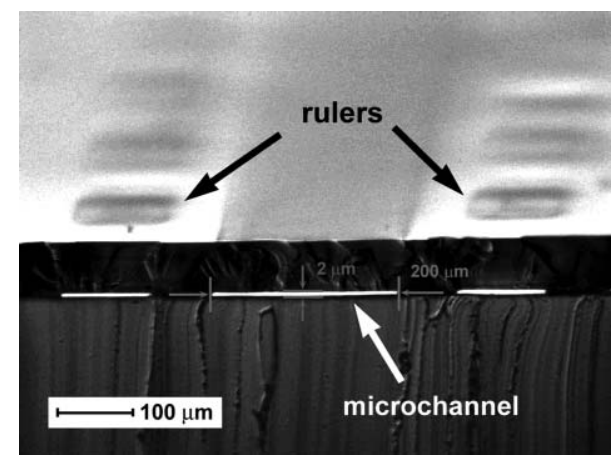

Fig. 2 Cross sectional microchannel view. The white areas in the section are the open channels. liquid volume through fluorescence detection. Furthermore, achieving $h / W$ values below 0.1 is an important prerequisite for realizing nanofluidic channels with micron-scale lateral resolution, which is accessible to standard photolithography fabrication. The completion of the PFPE slab curing by UV exposure of the whole chip after the superposition of the hybrid mold is crucial for faithfully preserving the original cross sectional dimensions of the polymer features, moreover irreversible sealing of the fluidic network and the cap.

Loss of sealing between the PFPE surfaces, finally causing the microchannel delamination, is typically observed at injection flow rates of $45 \mu \mathrm{min}^{-1}$ (Fig. 3). The results of the leakage experiments on the sealed devices are reported in Fig. 4, showing the measured average filling velocity $(v)$ of the injected liquid as a function of the externally applied flow rate. $v$ increases linearly from 2.4 to $54.0 \mathrm{~mm} \mathrm{~s}^{-1}$ upon increasing the applied flow rate, indicating watertight sealing. To better quantify this performance, we estimate the pressure gradient through the microchannels, $\Delta p / \Delta x$, where $\Delta p$ is the difference of pressure between the inlet and the outlet of a capillary of length, $\Delta x(1 \mathrm{~cm}$ for our samples). To this aim we use a modified Hagen-Poiseuille equation for laminar flow through capillaries with non-circular cross section: ${ }^{31}$

$$
J_{v}=-k \frac{\pi D_{\mathrm{h}}^{4}}{128 \mu} \frac{\Delta p}{\Delta x}
$$

where $J_{v}$ is the volume flow rate $(\cong W h v), D_{\mathrm{h}}$ is the channel hydraulic diameter $[2 W h /(W+h)], \mu$ is the water dynamic

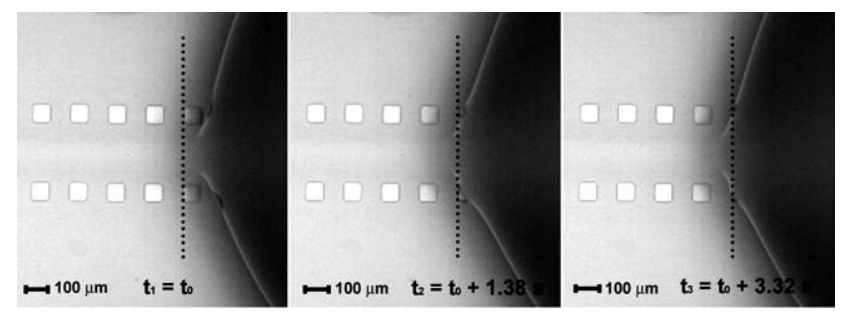

Fig. 3 Microchannel delamination starting (at time $t_{1}=t_{0}$ ) corresponding to the fluidic inlet, photographed at three different time instants while proceeding along the capillary axis. The dotted lines indicate the position (fixed) of the first ruler tick as a reference. Injection rate $=45 \mu \mathrm{min}^{-1}$. No delamination is observed at the PDMS/PFPE interface.

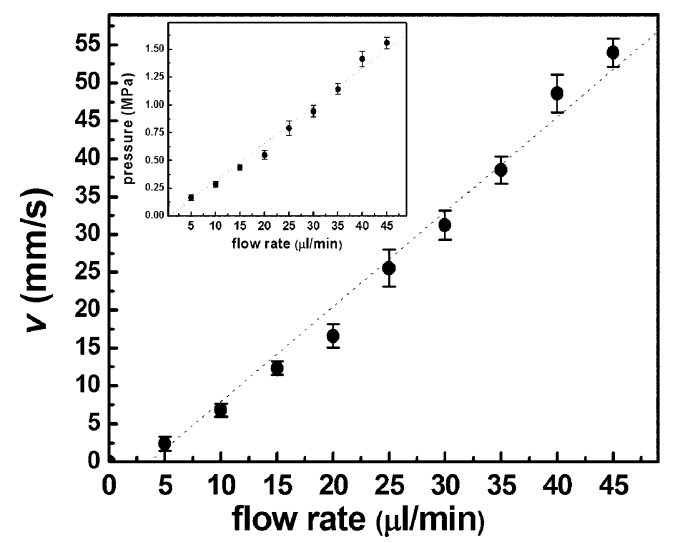

Fig. 4 Filling velocity $v s$. injection rate. The dotted line is a linear fit to the experimental data. Inset: inlet pressure $v s$. injection rate. The dotted line is a linear fit to experimental data. 
viscosity $\left(8.94 \times 10^{-4} \mathrm{~Pa} \mathrm{~s}\right)$, and $k$ is a constant depending on the conduct section shape ( $\cong 22$ for our geometry). ${ }^{31}$ The pressure at the channel entrance $\left(p_{i}\right)$ is calculated as:

$$
p_{i}=p_{\text {atm }}+\Delta p
$$

where $p_{\text {atm }}$ is the atmospheric pressure at the capillary outlet $(\cong 0.1 \mathrm{MPa})$. A maximum bond strength of $1.52 \mathrm{MPa}$ is achieved for our devices (inset of Fig. 4). For the sake of comparison, we recall that this value is higher, by a factor from 2 to 20, than typical values reported for plasma-treated or glued PDMS chips and interconnects. ${ }^{32-34}$

Different to previous methods developed for fully PFPEbased chips, ${ }^{26}$ this approach allows one to greatly reduce the fluoroelastomer waste during the fabrication of the mold textured with the microchannels, to decrease the overall curing times, and to exploit the more favorable conformability and elasticity properties of PDMS for the device handling and interconnection. No delamination was observed in our devices upon continuously injecting chloroform and toluene in the microchannels over two hours.

\section{Conclusions}

We realize hybrid PFPE-urethane-methacrylate/PDMS by a UV-based bonding approach, sealing the sidewalls of the microfluidic channels entirely by PFPE. The microchannels exhibit a very low aspect ratio (0.01) without capillary clogging or deformation. The delamination pressure $(1.52 \mathrm{MPa})$ at bonded interfaces is higher than other values for elastomeric materials. These disposable microfluidic devices can be exploited for many chemical and biological applications employing organic solvents.

\section{Acknowledgements}

The authors are grateful to the support of the Apulia Regional Strategic Project PS_144, and to Dr A. Zompatori at SolvaySolexis for kindly providing the PFPE elastomer.

\section{Notes and references}

1 D. A. Mair, M. Rolandi, M. Snauko, R. Noroski, F. Svec and J. M. J. Fréchet, Anal. Chem., 2007, 79, 5097-5102.

2 G. B. Lee, S. H. Chen, G. R. Huang, W. C. Sung and Y. H. Lin, Sens. Actuators, B, 2001, 75, 142-148.

3 S. Qi, X. Liu, S. Ford, J. Barrows, G. Thomas, K. Kelly, A. McCandless, K. Lian, J. Goettert and S. A. Soper, Lab Chip, 2002, 2, 88-95.
4 L. Brown, T. Koerner, J. H. Horton and R. D. Oleschuk, Lab Chip, 2006, 6, 66-73.

5 D. Day and M. Gu, Opt. Express, 2005, 13, 5939-5946.

6 Y. Sun, Y. C. Kwok and N. T. Nguyen, J. Micromech. Microeng., 2006, 16, 1681-1688.

7 G. Xu, J. Wang, Y. Chen, L. Zhang, D. Wang and G. Chen, Lab Chip, 2006, 6, 145-148.

8 J. Chen, Y. Lin and G. Chen, Electrophoresis, 2007, 28, 2897-2903.

9 D. S. Lee, H. Yang, K. H. Chung and H. B. Pyo, Anal. Chem., 2005, 77, 5414-5420.

10 D. A. Mair, E. Geiger, A. P. Pisano, J. M. J. Fréchet and F. Svec, $L a b$ Chip, 2006, 6, 1346-1354.

11 D. C. Duffy, J. C. McDonalds, O. J. A. Schueller and G. M. Whitesides, Anal. Chem., 1998, 70, 4974-4984.

12 J. C. McDonald, D. C. Duffy, J. R. Anderson, D. T. Chiu, H. Wu, O. J. A. Schueller and G. M. Whitesides, Electrophoresis, 2000, 21, $27-40$.

13 M. A. Unger, H. Chou, T. Thorsen, A. Scherer and S. R. Quake, Science, 2000, 288, 113-116.

14 G. M. Whitesides, Nature, 2006, 442, 368-373.

15 J. C. McDonald, S. J. Metallo and G. M. Whitesides, Anal. Chem., 2001, 73, 5645-5650.

16 X. Zhu, G. Liu, Y. Guo and Y. Tian, Microsyst. Technol., 2007, 13, 403-407.

17 J. Steigert, S. Haeberle, T. Brenner, C. Muller, C. P. Steinert, P. Koltay, N. Gottschlich, H. Reinecke, J. Ruhe, R. Zengerle and J. Ducrée, J. Micromech. Microeng., 2007, 17, 333-341.

18 Z. Chen, Y. Gao, J. Lin, R. Su and Y. Xie, J. Chromatogr., A, 2004, 1038, 239-245.

19 P. Abgrall, N. L. Low and N. T. Nguyen, Lab Chip, 2007, 7, 520 522.

20 C. W. Tsao, L. Hromada, J. Liu, P. Kumar and D. L. DeVoe, Lab Chip, 2007, 7, 499-505.

21 C. L. Lago, H. D. T. Silva, C. A. Neves, J. G. A. Brito-Neto and J. A. F. Silva, Anal. Chem., 2003, 75, 3853-3858.

22 A. Han, O. Wang, M. Graff, S. K. Mohanty, T. L. Edwards, K.-H. Han and A. B. Frazier, Lab Chip, 2003, 3, 150-157.

23 T. Nielsen, D. Nilsson, F. Bundgaard, P. Shi, P. Szabo, O. Geschke and A Kristensen, J. Vac. Sci. Technol., B, 2004, 22, 1770-1775.

24 J. N. Lee, C. Park and G. M. Whitesides, Anal. Chem., 2003, 75, 6544-6554.

25 C. H. Lin, C. H. Chao and C. W. Lan, Sens. Actuators, B, 2007, 121, 698-705.

26 J. P. Rolland, R. M. Van Dam, D. A. Schorzman, S. R. Quake and J. M. DeSimone, J. Am. Chem. Soc., 2004, 126, 2322-2323.

27 J. P. Rolland, E. C. Hagberg, G. M. Denison, K. M. Carter and J. M. De Simone, Angew. Chem., Int. Ed., 2004, 43, 5796-5799.

28 P. Fabbri, M. Messori, M. Montecchi, S. Nannarone, L. Pasquali, F. Pilati, C. Tonelli and M. Toselli, Polymer, 2006, 47, 1055-1060.

29 T. T. Truong, R. Lin, S. Jeon, H. H. Lee, J. Maria, A. Gaur, F. Hua, I. Meinel and J. A. Rogers, Langmuir, 2007, 23, 2898-2905.

30 E. Delamarche, H. Schmid, B. Michel and H. Biebuyck, Adv. Mater., 1997, 9, 741-746.

31 A. M. Lewis and E. R. Boose, Am. J. Bot., 1995, 82, 1112-1116.

$32 \mathrm{~S}$. Li and S. Chen, IEEE Trans. Adv. Packag., 2003, 26, 242-247.

33 A. Y. N. Hui, G. Wang, B. Linb and W. T. Chan, Lab Chip, 2005, 5, $1173-1177$.

34 B. Samel, M. K. Chowdhury and G. Stemme, J. Micromech. Microeng., 2007, 17, 1710-1714. 